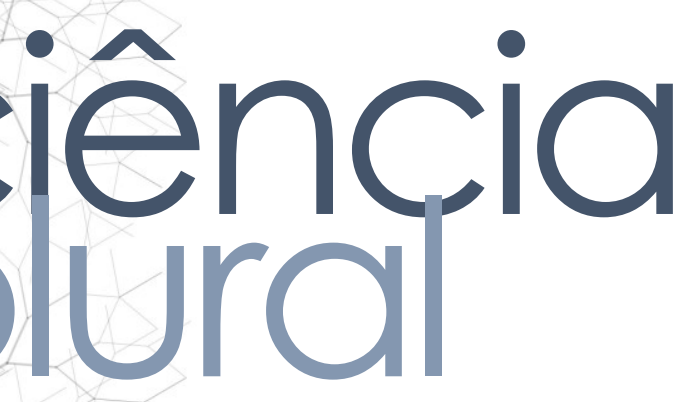

\title{
ACESSO AO SERVIÇO HOSPITALAR ORTOPÉDICO PÓS-TRAUMA NO ESTADO DO RIO GRANDE DO NORTE
}

Access to the post-trauma orthopedic hospital service in the state of Rio Grande do Norte

Acceso al servicio hospitalario ortopédico post-trauma en el estado de Rio Grande do Norte

Flávia Christiane de Azevedo Machado • Professora adjunta do Departamento de Saúde Coletiva da Universidade Federal do Rio Grande do Norte-UFRN • E-mail: flavitamachado@yahoo.com.br

Giselle Costa de Araújo Souza • Enfermeira e Sanitarista pela Universidade Federal do Rio Grande do Norte-UFRN • E-mail: giselle.ufrn@gmail.com

Autora responsável pela correspondência:

Flávia Christiane de Azevedo • E-mail: flavitamachado@yahoo.com.br 


\section{RESUMO}

Introdução: Existe um descompasso entre a oferta de serviços em ortopedia e a busca aos pronto-atendimentos por trauma. Objetivo: Assim, objetivou-se analisar o tempo entre o acesso ao serviço hospitalar ortopédico pós-trauma e a avaliação pelo ortopedista para os devidos encaminhamentos no Sistema Único de Saúde no Estado do Rio Grande do Norte de 2017 a 2018. Metodologia: Para tanto, desenvolveu-se uma investigação documental, na qual foram analisados, pelo cálculo das medidas de tendência central, dados do sistema de regulação estadual sobre os agendamentos para avaliação em ortopedia e sobre o perfil dos indivíduos que buscam o serviço. Resultados: No RN, em 2017, dos 499 registros na regulação em ortopedia, dois foram avaliados (0,4\%). Em 2018, de 688, 149 foram avaliados (21,6\%), com média de 67 dias de espera. Em Natal, no ano de 2017, o tempo médio de espera pela consulta foi de 119 dias e, em 2018, 22 dias. Conclusões: Verifica-se falha na oportunidade do cuidado, mediante o parâmetro de 5 a 10 dias após o trauma para os encaminhamentos.

Palavras-Chave: Regulação e fiscalização em saúde; Ortopedia; Traumatologia; Acesso aos Serviços de Saúde.

\section{ABSTRACT}

Introduction: There is a mismatch between the offer of services in orthopedics and the search in emergency rooms for trauma. Objective: Thus, the objective was to analyze the time between access to the post-trauma orthopedic hospital service and the evaluation by the orthopedist for the appropriate referrals to Unified Health System in the state of Rio Grande do Norte from 2017 to 2018. Methodology: To this end, a documentary investigation was developed, in which data from the state regulation system on the schedules for evaluation in orthopedics and on the profile of individuals seeking the service were analyzed by calculating the measures of central tendency. Results: In RN, in 2017, of the 499 records in orthopedics regulation, two were evaluated $(0.4 \%)$. In 2018, of 688,149 were evaluated $(21.6 \%)$, with an average of 67 days of waiting. In Natal, the average waiting time for consultation was 119 days and, in 2018, 22 days. Conclusions: There is a failure in the opportunity for care, using the parameter from 5 to 10 days after the trauma for referrals.

Keywords: Health Care Coordination and Monitoring; Orthopedics; Traumatology; Health Services Accessibility.

\section{ESUMEN}

troducción: Existe un desajuste entre la oferta de servicios en ortopedia y la úsqueda de traumatismos en las salas de emergencia. Objetivo: Por lo tanto, el bjetivo fue analizar el tiempo transcurrido entre el acceso al servicio hospitalario de opedia postraumática y la evaluación del ortopedista para referencias apropiadas el Sistema Único de Salud en el estado de Rio Grande do Norte de 2017 a 2018. 
Metodología: Para ello, se realizó una investigación documental, en la cual se analizaron, calculando medidas de tendencia central, datos del sistema de regulación estatal sobre los horarios de evaluación en ortopedia y sobre el perfil de las personas que buscan el servicio. Resultados: En RN, en 2017, de los 499 registros en regulación de ortopedia, se evaluaron dos (0.4\%). En 2018, de 688, 149 fueron evaluados (21.6\%), con un promedio de 67 días de espera. En Natal, el tiempo promedio de espera para la consulta fue de 119 días y, en 2018, de 22 días. Conclusiones: Hay una falla en la oportunidad de atención, utilizando el parámetro de 5 a 10 días después del trauma para las derivaciones.

Palabras clave: Regulación y Fiscalización en salud; Ortopedia; Traumatología; Accesibilidad a los Servicios de Salud. 


\section{Introdução}

O Sistema Único de Saúde (SUS) viabiliza acesso gratuito e regulado através de estratégias como protocolos e classificação de risco a todo cidadão ${ }^{1,2}$. Assim, busca-se a equidade no acesso para organizar, controlar, gerenciar e priorizar o cuidado de modo a equilibrar a necessidade-demanda-oferta, tornando-a coerente e sem grandes distorções ${ }^{3}$. Todavia, as limitações de recursos dispendidos pelo Estado Brasileiro têm repercutido no fenômeno da judicialização, onde o Poder Judiciário determina concretização do direito à saúde pela imposição ao Poder Público4.

As demandas da judicialização envolvem fornecimento de medicamentos, realização de cirurgias, procedimentos e até incorporação de novas tecnologias no âmbito do SUS. Por excederem a capacidade produtiva, tais demandas preocupam gestores da saúde em todos os níveis federativos ${ }^{5}$. De acordo com Biehl ${ }^{6}$, os pacientescidadãos-consumidores não mais esperam que as tecnologias médicas se redistribuam, e a judicialização se tornou um instrumento essencial para a responsabilização do Estado por infraestruturas eficazes.

A "Ortopedia e Traumatologia"7 é uma especialidade que unifica a ortopedia, relacionada a doenças e deformidades do aparelho locomotor (ossos, músculos, ligamentos e articulações) e a traumatologia, que lida com o trauma do aparelho musculoesquelético. Os tratamentos da traumatologia podem ser conservadores (ligaduras, imobilização com gesso) e invasivos (intervenções cirúrgicas para instalar parafusos, placas e outros elementos no interior do corpo) a depender do tipo de lesão7.

A crescente violência, doenças ocupacionais, acidentes de trânsito e causas externas têm repercutido em demandas da traumatologia com marcantes impactos orçamentários e na morbimortalidade, incluindo o contexto da judicialização. Segundo a Sociedade Brasileira de Ortopedia e Traumatologia (SBOT) ${ }^{8}$, o politrauma a principal causa de morte na faixa etária entre cinco e 44 anos. As lesões que ometem o politraumatizado, quando não levam ao óbito, deixam sequelas rmanentes, culminando em elevados custos pessoais e psicológicos aos familiares e edade. 
Mediante um politrauma, primeiro objetiva-se a sobrevivência com funções cognitivas normais e otimizado o estado geral do paciente, deve ocorrer o tratamento definitivo. Este é o conceito do controle de danos, com o objetivo de salvar a vida, adiando o reparo definitivo das lesões. Isto porque o tratamento imediato aumenta a reação inflamatória sistêmica, podendo levar ao óbito. Assim, após o controle de danos, a cirurgia definitiva das fraturas dos ossos longos deve ser agendada dentro da “janela imunológica de oportunidade", período entre o quinto e o décimo dia pós trauma ${ }^{8}$.

Não obstante, estudos mostram que a estratégia de cirurgia precoce para fratura do quadril em idosos apresenta menor custo e melhores resultados em relação à tardia (mais de quatro dias entre o trauma e a cirurgia) ${ }^{9}$. Portanto, percebe-se a importância de uma intervenção cirúrgica em tempo oportuno para uma atenção à saúde de qualidade.

Neste sentido, iniciativas como o Projeto Suporte em Ortopedia e Traumatologia, criado pelo Instituto Nacional de Tráumato-Ortopedia (Into), no contexto da atenção especializada no SUS, busca promover a estruturação de serviços de tráumato-ortopedia e de reabilitação pós-operatória, por meio do apoio técnico e financeiro às Secretarias Estaduais e Municipais de Saúde ${ }^{10}$.

Portanto, o descompasso entre a demanda crescente pelo pronto-atendimento por trauma e a limitação na oferta de serviços em ortopedia, repercutindo em ações judiciais ao ente público, motivaram a realização deste estudo que objetivou analisar o tempo entre o acesso ao serviço hospitalar (urgência) ortopédico pós-trauma e a avaliação pelo especialista ortopedista para os devidos encaminhamentos (cirurgia) no SUS no estado do Rio Grande do Norte-RN de 2017 a 2018.

\section{Metodologia}

Pesquisa quantitativa do tipo descritiva, retrospectiva, transversal, com ratégia de investigação documental, onde foram analisados dados do sistema de ulação sobre os agendamentos para avaliação em ortopedia no estado do RN no íodo de 2017 a 2018. No caso, a medida desfecho analisada foi o tempo transcorrido de o acesso ao serviço hospitalar no pronto-atendimento (urgência) ortopédico pós- 
trauma e a avaliação pelo especialista ortopedista para os devidos encaminhamentos (reparo cirúrgico). Para isso, foi construída uma tabela no Excel®. A hipótese é que o tempo útil para o desfecho nas avaliações em ortopedia não esteja dentro da janela imunológica de oportunidade, entre o quinto e décimo dia pós-trauma.

Além das informações sobre o período de tempo, pretendeu-se identificar o tipo de tratamento ortopédico que prevalece nas demandas e o perfil dos indivíduos (sexo, idade, município de proveniência, parte do corpo acometida). Assim, foram adotados os seguintes critérios de inclusão: fratura ocorrida nos anos de 2017 e 2018, presença de informações sobre o perfil. Foram excluídos do estudo dados de pacientes com informações incompletas, fundamentais para os parâmetros.

Os dados referentes ao tempo de espera para a consulta com o especialista foram analisados pelas medidas de tendência central com auxílio do Programa PSPP®. Por sua vez, as variáveis categóricas foram analisadas pelas frequências absolutas e relativas.

Em meio ao risco de, ocasionalmente, haver exposição do histórico dos pacientes durante a coleta de informações este estudo teve o protocolo submetido à apreciação ética do CEP/HUOL/UFRN, obtendo parecer favorável número 3.215.948.

Em 2019, os Hospitais credenciados em alta complexidade para traumas ortopédicos no RN localizados na capital do estado, em Natal, eram: Hospital Memorial, Hospital Municipal Doutor Newton Azevedo, Hospital Doutor Ruy Pereira dos Santos e o Hospital Monsenhor Walfredo Gurgel, sendo que apenas o Memorial encontra-se habilitado pelo Ministério da Saúde, apesar dos demais haverem enviado documentação para habilitação.

Os procedimentos não possíveis de execução no próprio Estado são encaminhados para tratamento fora de domicílio, através da Central Nacional de Regulamentação de Alta Complexidade - CNRAC.

As informações que subsidiam este estudo foram captadas em abril de 2019 no ácleo de Ortopedia, localizado no Complexo Estadual de Regulação, que é o setor Secretaria de Estado da Saúde Pública (SESAP) responsável pela coordenação dos intos relacionados ao tema no estado. As informações foram enviadas através de 
tabelas com os dados, que foram trabalhados em formato de planilha condensando as variáveis de interesse. As análises foram realizadas sob um recorte estadual, fornecido pelo Hospital Monsenhor Walfredo Gurgel - HMWG, abarcando um público proveniente dos vários municípios do estado, e um recorte da capital, enviado pelo Hospital Municipal de Natal - HMN. Os dados foram do período de 2017 e 2018, pois antes desse período, o Núcleo de Ortopedia não armazenava as informações.

O Núcleo de Ortopedia recebe de 15 a 20 casos por dia para agendar avaliação em no máximo uma semana. Os casos de fraturas até 30 dias de tornozelo e pé em adultos podem ser agendados no Hospital Memorial, com vaga para 160 consultas mensais. Os casos de fraturas até 30 dias de tornozelo, pé, tíbia, antebraço e punho em adultos podem ser agendados no Hospital Rui Pereira, com vaga para 40 consultas mensais. Todos os tipos de fratura, com prioridade para clavícula, com mais de 30 dias, para pacientes de até 60 anos são agendados para a clínica Paulo Gurgel, com vaga para 48 consultas mensais. Esse agendamento é realizado de acordo com a oferta de vagas na rede, estando todos numa mesma lista de espera.

\section{Resultados}

No período estudado, algumas cirurgias estavam interrompidas por falta de material e sem previsão de retorno. Isto, por si, revela uma grande fragilidade no acesso a essas cirurgias. Todavia, partindo para análise das informações alvo deste estudo, no tocante ao recorte estadual.

Em 2017, haviam 488 pacientes registrados na regulação em ortopedia, dos quais apenas dois foram avaliados $(0,4 \%)$ e 11 receberam alta ainda no primeiro atendimento (sendo um a pedido do paciente), permanecendo $475(97,4 \%)$ pacientes aguardando avaliação.

Em 2018, de um total de 688 pacientes, 149 foram avaliados (21,6\%), 12 eceberam alta (sendo três a pedido do paciente, quatro não precisavam da cirurgia ou ão quiseram) e 527 (76,6\%) ficaram aguardando. Apenas um informou tê-la realizado articular. Dessa forma, o número de agendamentos em 2017 foi expressivamente nor, possivelmente, por ser o período de suspensão das cirurgias. 
Quanto ao tempo de espera pelo atendimento, os dois pacientes do ano de 2017 esperaram 185 e 227 dias, sendo atendidos no ano seguinte. Em 2018, o tempo de espera variou de 1 a 134 dias, sendo todos atendidos no mesmo ano, perfazendo uma média de 67 dias de espera.

Nos dois anos analisados, observa-se que o número de pacientes do sexo masculino é cerca de 3 vezes maior que o número de pacientes do sexo feminino (75 e 72\% do sexo masculino em 2017 e 2018, respectivamente).

Pacientes na faixa etária de 20 a 39 anos corresponde a cerca de 50\% do total, em ambos os anos, tanto avaliados como esperando por consulta. Números maiores também são visualizados em pacientes com mais de 60 anos de idade (Tabela 1).

Em relação à procedência, a maioria dos pacientes é proveniente de Natal, seguida de Parnamirim, São Gonçalo do Amarante e Macaíba. Tais municípios são os mais populosos do estado, sendo este um resultado esperado.

Quanto à parte do corpo acometida no trauma, a clavícula está predominante, seguida do tornozelo e úmero nos dois anos estudados, seguidos de pé (2017) e mão (2018), como mostra a Tabela 2.

No recorte municipal, em 2017, 25 dos 37 pacientes foram avaliados, ou seja, 67,6\%. Em 2018, 198 (46,7\%) pacientes foram avaliados, 8 não precisaram ou não se conseguiu contato e 218 permaneceram esperando, de um total de 424 . Apenas um já havia realizado particular.

O tempo de espera pela consulta variou em 2017 de 2 a 516 dias e, em 2018, de 1 a 157 dias, perfazendo médias de 119 e 22 dias, respectivamente, conforme Figura 1.

Na capital, mantém-se a prevalência de pacientes do sexo masculino (73,0 e $63,7 \%$ nos dois anos). Cerca de $40 \%$ dos pacientes tem entre 20 e 39 anos de idade, como mostra a Tabela 1. 
Figura 1 - Quantidade de dias de espera dos pacientes de traumas ortopédicos, no estado do Rio Grande do Norte e na capital, no período de 2017 a 2018. Natal/RN, 2019.

\section{Dias de espera}

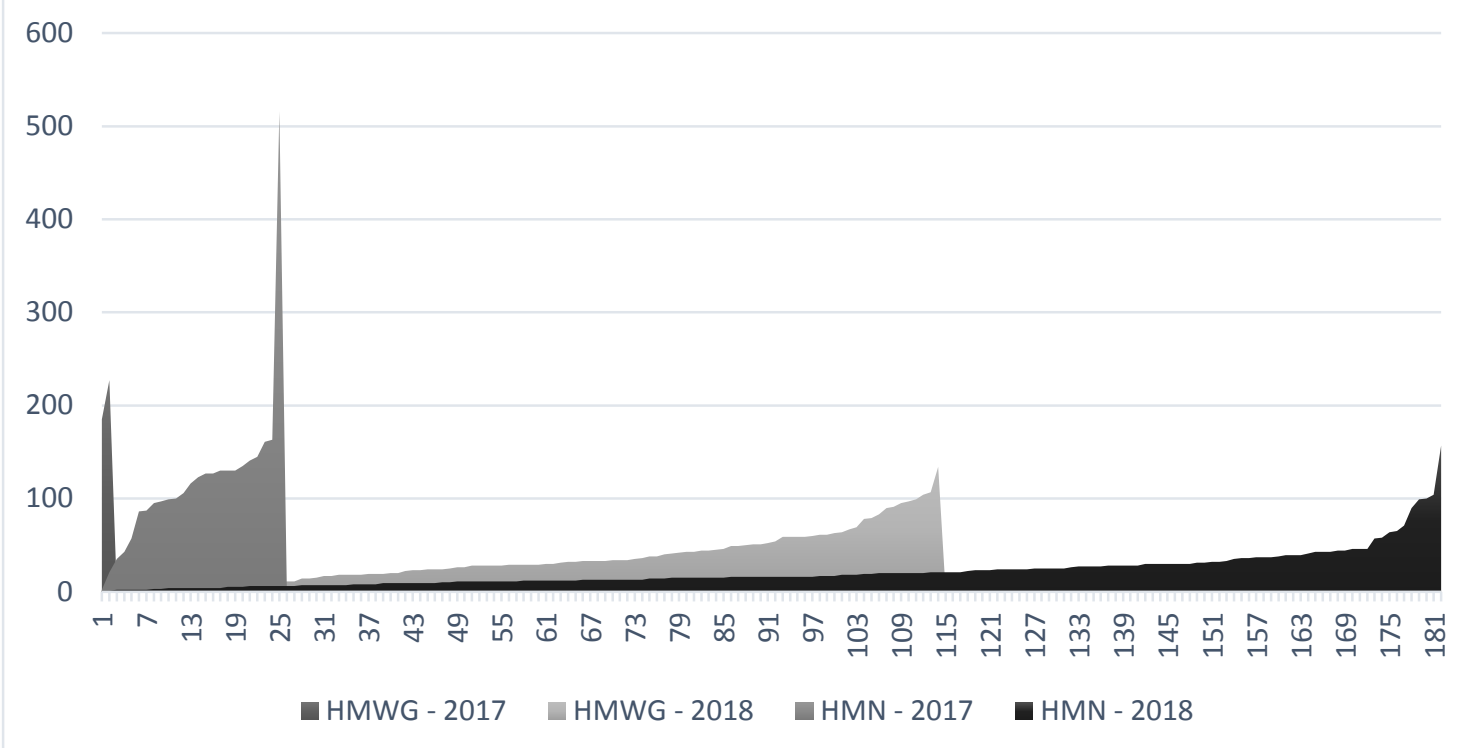

Legenda:

HMWG: Hospital Monsenhor Wafredo Gurgel

HMN: Hospital Municipal de Natal

Tabela 1 - Distribuição dos pacientes de traumas ortopédicos por faixas etárias, no estado do RN e na capital, no período de 2017 a 2018. Natal-RN/2019.

\begin{tabular}{ccccc}
\hline Estado do $R N$ & \multicolumn{2}{c}{2017} & \multicolumn{2}{c}{2018} \\
Idade & avaliados & esperando & avaliados & esperando \\
\hline $\mathbf{1 0}$ a $\mathbf{1 9}$ & 0 & 44 & 15 & 35 \\
\hline $\mathbf{2 0} \boldsymbol{a} \mathbf{2 9}$ & 0 & 122 & 44 & 120 \\
\hline $\mathbf{3 0} \boldsymbol{a} \mathbf{3 9}$ & 0 & 118 & 29 & 103 \\
\hline $\mathbf{4 0} \boldsymbol{a} \mathbf{4 9}$ & 0 & 73 & 24 & 88 \\
\hline $\mathbf{5 0} \boldsymbol{a} \mathbf{5 9}$ & 0 & 72 & 24 & 66 \\
\hline$>\mathbf{6 0}$ & 2 & 46 & 13 & 115 \\
\hline Total & $\mathbf{2}$ & $\mathbf{4 7 5}$ & $\mathbf{1 4 9}$ & $\mathbf{5 2 7}$ \\
\hline Capital & \multicolumn{2}{c}{$\mathbf{2 0 1 7}$} & \multicolumn{3}{c}{$\mathbf{2 0 1 8}$} \\
\hline Idade & avaliados & $\mathbf{e s p e r a n d o}$ & avaliados & Esperando \\
\hline $\mathbf{1 0} \boldsymbol{a} \mathbf{1 9}$ & 2 & 0 & 12 & 23 \\
\hline $\mathbf{2 0} \boldsymbol{a} \mathbf{2 9}$ & 3 & 2 & 53 & 34 \\
\hline $\mathbf{3 0} \boldsymbol{a} \mathbf{3 9}$ & 9 & 1 & 34 & 46 \\
\hline $\mathbf{4 0} \boldsymbol{a} \mathbf{4 9}$ & 4 & 4 & 35 & 31 \\
\hline $\mathbf{5 0} \boldsymbol{a} \mathbf{5 9}$ & 5 & 2 & 31 & 32 \\
\hline$>\mathbf{6 0}$ & 2 & 3 & 33 & 52 \\
\hline Total & $\mathbf{2 5}$ & $\mathbf{1 2}$ & $\mathbf{1 9 8}$ & $\mathbf{2 1 8}$ \\
\hline
\end{tabular}




\section{cjência}

As partes do corpo mais acometidas são mão, tornozelo, punho, rádio e clavícula, nessa ordem decrescente, que também são as partes que mais receberam avaliação médica, conforme Tabela 2.

Tabela 2 - Partes do corpo acometidas no estado do RN e na capital, no período de 2017 a 2018. Natal-RN/2019.

\begin{tabular}{|c|c|c|c|c|}
\hline Estado do RN & \multicolumn{2}{|c|}{2017} & \multicolumn{2}{|c|}{2018} \\
\hline Partes do corpo & avaliados & esperando & avaliados & esperando \\
\hline CALCÂNEO & 0 & 11 & 2 & 39 \\
\hline CLAVÍCULA & 0 & 3 & 2 & 19 \\
\hline COTOVELO & 0 & 1 & 4 & 4 \\
\hline FÊMUR & 1 & 1 & 5 & 2 \\
\hline HÁLUX & 0 & 4 & 7 & 37 \\
\hline JOELHO & 0 & 0 & 23 & 16 \\
\hline$M \tilde{A} O$ & 0 & 0 & 9 & 8 \\
\hline OMBRO & 0 & 6 & 26 & 19 \\
\hline$P E$ & 0 & 7 & 23 & 12 \\
\hline PERNA & 0 & 11 & 28 & 27 \\
\hline PLATÔ TIBIAL & 0 & 25 & 27 & 53 \\
\hline PUNHO & 0 & 3 & 22 & 15 \\
\hline QUADRIL & 0 & 8 & 46 & 23 \\
\hline RÁDIO & 1 & 11 & 71 & 115 \\
\hline TORNOZELO & 0 & 22 & 87 & 52 \\
\hline ULNA & 0 & 34 & 103 & 76 \\
\hline ÚMERO & 0 & 0 & 0 & 4 \\
\hline Total & 2 & 147 & 485 & 517 \\
\hline Capital & \multicolumn{2}{|c|}{2017} & \multicolumn{2}{|c|}{2018} \\
\hline Partes do corpo & avaliados & esperando & avaliados & Esperando \\
\hline CALCÂNEO & 1 & 9 & 0 & 7 \\
\hline CLAVÍCULA & 4 & 12 & 0 & 19 \\
\hline COTOVELO & 0 & 0 & 0 & 0 \\
\hline FÊMUR & 0 & 0 & 0 & 2 \\
\hline HÁLUX & 1 & 3 & 0 & 4 \\
\hline JOELHO & 2 & 1 & 0 & 17 \\
\hline$M \tilde{A} O$ & 4 & 61 & 3 & 25 \\
\hline OMBRO & 1 & 6 & 3 & 11 \\
\hline$P E$ & 2 & 5 & 0 & 14 \\
\hline PERNA & 0 & 0 & 0 & 7 \\
\hline PLATÔ TIBIAL & 0 & 0 & 0 & 0 \\
\hline PUNHO & 1 & 34 & 2 & 13 \\
\hline QUADRIL & 2 & 0 & 1 & 1 \\
\hline RÁDIO & 3 & 20 & 0 & 20 \\
\hline
\end{tabular}




\begin{tabular}{ccccc}
\hline TORNOZELO & 3 & 35 & 2 & 26 \\
\hline ULNA & 1 & 6 & 0 & 4 \\
\hline ÚMERO & 0 & 10 & 1 & 29 \\
\hline Total & $\mathbf{2 5}$ & $\mathbf{2 0 2}$ & $\mathbf{1 2}$ & $\mathbf{1 9 9}$ \\
\hline
\end{tabular}

\section{Discussão}

Assim como nos estudos analisados mais de $90 \%$ dos tratamentos para vítimas de traumas são abordagens cirúrgicas, no presente estudo analisamos uma relação de pacientes à espera por tratamento cirúrgico. Verifica-se um tempo de espera bastante longo entre os que conseguiram agendamento da avaliação médica, sem mencionar os que continuam aguardando.

O estudo mostra uma notória falha na oportunidade do cuidado, uma vez que encontramos um tempo muito superior ao ideal para o devido atendimento, com períodos que se contrapõem com o parâmetro da "janela imunológica de oportunidade", entre o quinto e o décimo dia pós trauma ${ }^{8}$. Assim, as sequelas podem ser irreparáveis, o procedimento mais complicado e mais dispendioso. Esse dado aponta a necessidade de serviços de atendimento preparados para uma abordagem multidisciplinar.

De acordo com Maurício Júnior, Presidente da Sociedade Brasileira de Trauma Ortopédico, na região Nordeste é grande a insatisfação dos profissionais dedicados ao trauma ortopédico, especialmente nos hospitais públicos. A falta de infraestrutura mínima de trabalho faz com que pacientes sejam atendidos e tratados muito tardiamente, muitos deles após a consolidação de suas fraturas originais. Na rede privada são raros os hospitais de "porta aberta" ao atendimento do politraumatizado por julgarem ser este tipo de atendimento deficitário do ponto de vista econômico, além da dificuldade para a autorização de procedimentos ${ }^{14}$.

Estudo realizado por Santos ${ }^{11}$, em serviço público de emergência a vítimas de trauma no Piauí, evidenciou como tempo médio de internação o intervalo de 1 a 15 dias, abrangendo $89,4 \%$ dos casos, considerado um tempo médio equiparado a outros udos. 


\section{ciênncia
pural}

O paciente do trauma ortopédico na rede pública convive com a seguinte realidade: como o seu mal em geral não se associa ao óbito, seu atendimento tende a ser diferido para momentos onde não existam outras urgências consideradas "maiores". O resultado disso é o atendimento tardio de fraturas expostas, com muito mais do que seis horas decorridas desde o trauma, ou um tempo médio de espera de 3 a 7 dias para o tratamento de uma fratura trocantérica de fêmur. A sequela resultante de um atendimento diferido não é objetivamente quantificada em nosso país, mas há inúmeras evidências internacionais que o tratamento imediato do trauma ortopédico, seja para uma conduta definitiva, seja para um controle de danos ortopédicos, é o melhor que se pode oferecer ao paciente ${ }^{14}$.

Estudo alemão mostrou a incidência de complicações em pacientes idosos com fratura de quadril, sendo as mais comuns: delirium, anemia, infecções do trato urinário associadas ao cateter e pneumonia. ${ }^{15} \mathrm{Na}$ França, estudo retrospectivo com pacientes ortopédicos constatou uma média de tempo de internação de 12,6 dias, e necessidade de cirurgia ortopédica em $54,5 \%$ dos pacientes $^{16}$.

Uma das limitações do nosso estudo é o banco de dados não fornecer informações referentes à realização das cirurgias, que seriam relacionadas ao desfecho, uma vez que um dos objetivos do Núcleo de Ortopedia é possibilitar a avaliação médica com especialista antes da cirurgia aos indivíduos que aguardam em sua residência. Eventualmente, nesse processo da busca ativa do indivíduo podem ocorrer três desfechos: informe que já fez a cirurgia, que não precisa dela ou que faz outro tratamento como, por exemplo, a fisioterapia. No caso, as demandas por cirurgias ortopédicas são inferidas pelo público que ainda aguarda, consequentemente, o desfecho de interesse é o número de agendamentos das avaliações de ortopedia.

Em relação as causas dos traumas, embora, não haja esta identificação nos bancos consultados, é possível inferir sobre os acidentes de trânsito com motocicletas partir da análise dos órgãos do corpo em que os traumas são mais frequentes. Isto tá em concordância a estudos que apontam a predominância de acidentes de ânsito, nos quais, em geral, os membros são os segmentos corpóreos mais atingidos, 
visto serem as regiões mais desprotegidas, com predominância dos membros superiores ${ }^{11,12,13}$.

Na literatura, encontra-se unanimidade na predominância de traumas em indivíduos do sexo masculino, com proporções semelhantes de cerca de 70\%, haja vista ser um grupo que se expõe mais a riscos, como desrespeito às leis de trânsito, uso de álcool e drogas, além de maior vulnerabilidade à violência urbana11,12,13 .

A maior participação de indivíduos jovens também foi observada em outros estudos relacionados ao trauma ortopédico, prevalecendo a faixa etária de 18-38, 1959, 20-39 anos.

O serviço público de saúde geralmente não dispõe de todos os procedimentos de média e alta complexidade compatíveis com a demanda, gerando um atraso na resolução dos problemas de saúde e determinando também a contratação de prestadores de serviços. Assim, infere-se sobre uma falha quanto à oportunidade do cuidado em vista do grande tempo transcorrido para os agendamentos das cirurgias. Esse estudo suscita, portanto, a importância e necessidade de se investir na oferta de serviço hospitalar ortopédico pós-trauma de qualidade para evitar maiores gastos futuros com saúde e aumento de morbidades pelo atraso no tratamento.

\section{Conclusões}

Houve comprovação da hipótese de que as avaliações em ortopedia não têm ocorrido em tempo útil dentro da janela imunológica de oportunidade; entre o quinto e décimo dia pós trauma. Espera-se que esse tipo de pesquisa possa subsidiar gestores locais do SUS nas ações de regulação, controle, avaliação e auditoria na atenção em Traumatologia e Ortopedia, inclusive em estudos de qualidade e de custo-efetividade.

Essa cobertura assistencial necessária, composta por demanda reprimida, culmina ainda no planejamento de estratégias conjuntas à (política de) prevenção de cidentes, os maiores responsáveis pelos atendimentos em traumas ortopédicos, que eram efeitos deletérios para qualidade de vida dos pacientes. Além disso, torna-se portante ressaltar a pertinência do controle social e auditoria diante de prestação de viços e contratos para o devido cumprimento dos acordos firmados. 


\section{Referências}

1. Brasil. Ministério da Saúde. SUS democratiza o acesso do cidadão aos serviços de saúde. Brasília. 2009. Disponível em: http://www.brasil.gov.br/saude/2009/11/sus-democratiza-o-acesso-do-cidadaoaos-servicos-de-saude

2. Brasil. Portaria $n^{\circ} 1.559$, de $1^{\circ}$ de agosto de 2008. Institui a Política Nacional de Regulação do Sistema Único de Saúde - SUS. Ministério da Saúde; 2008. Disponível em: http://bvsms.saude.gov.br/bvs/saudelegis/gm/2008/prt1559_01_08_2008.html

3. Vilarins GCM, Shimizui HE, Gutierrez, MMU. A regulação em saúde: aspectos conceituais e operacionais. Saúde em Debate. 2012; 36 (95): 640-647.

4. Silva, LMA. Judicialização do direito à saúde: aspectos relevantes no Direito Brasileiro. In: Âmbito Jurídico, Rio Grande, XIX, n. 144, jan 2016. Disponível em: http://ambitojuridico.com.br/site/index.php?n_link=revista_artigos_leitura\&artigo _id $=16728 \&$ revista_caderno $=9$

5. Brasil, Advocacia Geral da União, Consultoria Jurídica/ Ministério da Saúde. Intervenção Judicial na saúde pública. 2012. Disponível em: http:// portalarquivos.saude.gov.br/images/pdf/2014/maio/29/Panorama-dajudicializa----o---2012---modificado-em-junho-de-2013.pdf

6. Biehl J. Pacientes-cidadãos-consumidores: a judicialização da saúde e a metamorfose da biopolítica. Lua Nova [online]. 2016; (98): 77-105.

7. Brasil, Ministério da Saúde. Atenção Especializada e Hospitalar. Especialidades > Traumatologia e Ortopedia. Afeç̧ões Osteomusculares/ Músculo Esquelético. 2018. Disponível em: http://portalms.saude.gov.br/atencao-especializada-ehospitalar/especialidades/traumatologia-e-ortopedia/afeccoes-osteomuscularesmusculo-esqueletico

8. Sociedade Brasileira de Ortopedia e Traumatologia (SBOT). Manual de trauma ortopédico. Pozzi I, Reginaldo S, Almeida MV, Cristante AF (coordenadores). São Paulo: SBOT; 2011. Disponível em: https://sbot.org.br/wpcontent/uploads/2018/09/manual_trauma_ortopedico.pdf

Loures FB, Chaoubah A, Oliveira VM, Almeida AM, Campos SEM, Paiva EP. Análise econômica do tratamento cirúrgico de fratura do quadril em idosos. Rev Saúde tublica 2015; 49:12.

Brasil, Ministério da Saúde. Secretaria de Atenção à Saúde. Portaria de nsolidação $\mathrm{n}^{\circ}$ 5, de 28 de setembro de 2017. Disponível em: p://bvsms.saude.gov.br/bvs/saudelegis/gm/2017/prc0005_03_10_2017.html 
11. Santos LFS, Fonseca JMA, Cavalcante BLS, Lima CM. Estudo epidemiológico do trauma ortopédico em um serviço público de emergência. Cad. Saúde Colet. 2016; 24 (4): 397-403.

12. Sousa LRB, Sousa GS, Monroe KCMC, Ferreira MGS. Notificação do acidente traumático em um hospital público da Amazônia brasileira. Rev Bras Promoç Saúde. 2017; 30(1): 64-71.

13. Hildebrand CR. Fatores associados ao encaminhamento hospitalar de vítimas de trauma ortopédico atendidas no serviço de referência municipal em ortopediaCampo Grande/MS - 2009. 2010. 80 f. Dissertação (Saúde Pública) - Escola Nacional de Saúde Pública Sergio Arouca, Rio de Janeiro, 2010.

14. Kfuri Júnior M. O Trauma Ortopédico no Brasil. Rev Bras Ortop. 2011; 46 (supl.1).

15. Folbert E C, Hegeman J H, Gierveld R, Netten J J van, Velde D van der, Ten Duis $\mathrm{HJ}$, et al. Complications during hospitalization and risk factors in elderly patients with hip fracture following integrated orthogeriatric treatment. Arch Orthop Trauma Surg. 2017; 137(4):507-515.

16. Donnally CJ, Rothenberg PM, Metser G, Massel DH, Butler AJ, Damodar D, et al. Orthopedic injuries associated with jet-skis (personal watercrafts): A review of 127 inpatients. Orthop Traumatol Surg Res . 2018; 104(2):267-271. 